\title{
Ultrafast Oxidative Desulfurization of Diesel Fuels by Mass Transfer Enhancement of Polyoxometalate Modified Alumina \\ Catalysts
}

Yubing Liu ${ }^{\mathrm{a}, \uparrow}$, Jinfeng Chu ${ }^{\mathrm{a}, \dagger}$, Lifei Lian ${ }^{\mathrm{a}}$, Xiang Chen ${ }^{\mathrm{a}}$, Sai An ${ }^{\mathrm{a}}$, Lanlan Hong ${ }^{\mathrm{a}}$, Dongqi Wang*b and Wei Chen*a

${ }^{a}$ State Key Laboratory of Chemical Resource Engineering, Beijing Advanced Innovation Center for Soft Matter Science and Engineering, Beijing University of Chemical Technology, Beijing 100029, People's Republic of China

${ }^{b}$ State Key Laboratory of Fine Chemicals, Liaoning Key Laboratory for Catalytic Conversion of Carbon Resources, School of Chemical Engineering, Dalian University of Technology, Dalian 116024, People's Republic of China

*Email: chenw@mail.buct.edu.cn; wangdq@dlut.edu.cn; Tel/Fax: +86 10-64431832.

$\dagger$ These authors contributed equally on this work. 


\section{This file includes:}

Table S1. Characteristics of the Simulated Systems.

Figure S1. FT-IR spectra of $\mathrm{Al}_{2} \mathrm{O}_{3}, \mathrm{P}_{2} \mathrm{~W}_{15}, \mathrm{Al}_{2} \mathrm{O}_{3}-\mathrm{P}_{2} \mathrm{~W}_{15}$ and $\mathrm{Al}_{2} \mathrm{O}_{3}-\mathrm{P}_{2} \mathrm{~W}_{15}-\mathrm{C}_{18}$.

Figure S2. FT-IR spectra of $\mathrm{Al}_{2} \mathrm{O}_{3}-\mathrm{P}_{2} \mathrm{~W}_{15}-\mathrm{C}_{8}, \mathrm{Al}_{2} \mathrm{O}_{3}-\mathrm{P}_{2} \mathrm{~W}_{15}-\mathrm{C}_{12}$ and $\mathrm{Al}_{2} \mathrm{O}_{3}-\mathrm{P}_{2} \mathrm{~W}_{15}-\mathrm{C}_{18}$.

Figure S3. (a) SEM image of $\mathrm{Al}_{2} \mathrm{O}_{3}-\mathrm{P}_{2} \mathrm{~W}_{15}-\mathrm{C}_{8}$; (b) SEM image of $\mathrm{Al}_{2} \mathrm{O}_{3}-\mathrm{P}_{2} \mathrm{~W}_{15}-\mathrm{C}_{12}$; (c) TEM image of $\mathrm{Al}_{2} \mathrm{O}_{3}-\mathrm{P}_{2} \mathrm{~W}_{15}-\mathrm{C}_{8}$; (d) TEM image of $\mathrm{Al}_{2} \mathrm{O}_{3}-\mathrm{P}_{2} \mathrm{~W}_{15}-\mathrm{C}_{12}$.

Figure S4. SEM-EDS of $\mathrm{Al}_{2} \mathrm{O}_{3}-\mathrm{P}_{2} \mathrm{~W}_{15}-\mathrm{C}_{18}$.

Table S2. Textural properties of $\gamma-\mathrm{Al}_{2} \mathrm{O}_{3}, \mathrm{Al}_{2} \mathrm{O}_{3}-\mathrm{P}_{2} \mathrm{~W}_{15}$ and $\mathrm{Al}_{2} \mathrm{O}_{3}-\mathrm{P}_{2} \mathrm{~W}_{15}-\mathrm{C}_{18}$ catalysts.

Figure S5. (a) Effect of temperature on sulfur removal of DBT; (b) Effect of the amount of catalyst on sulfur removal of DBT.

Figure S6. The influence of reaction time on DBT removal measured by GC-FID analysis. Figure S7. (a) Effect of temperature on sulfur removal of diesel fuel; (b) Effect of the amount of $\mathrm{H}_{2} \mathrm{O}_{2}$ on sulfur removal of diesel fuel.

Table S3. Comparison of catalytic oxidative desulfurization of real diesel and gasoline fuels by different immobilized catalytic systems (reported in literature) and the $\mathrm{Al}_{2} \mathrm{O}_{3}$ $\mathrm{P}_{2} \mathrm{~W}_{15}-\mathrm{C}_{18}$ from the present study.

Figure S8. The image of solubility about $\mathrm{DBT}$ and $\mathrm{DBTO}_{2}$ in octadecyltrichlorosilane. 
Table S1. Characteristics of the Simulated Systems.

\begin{tabular}{|c|c|c|c|}
\hline Solute & Solvent & Box Size $(\AA)$ & Time (ns) \\
\hline \multirow{2}{*}{ 1DBT } & 300 Octane $\left(27 / 60^{\circ} \mathrm{C}\right)$ & $43.7 / 44.3$ & \multirow{4}{*}{2} \\
\hline & 2967Water $\left(27^{\circ} \mathrm{C}\right)$ & 44.7 & \\
\hline \multirow{2}{*}{$1 \mathrm{DBTO}_{2}$} & 300 Octane $\left(27 / 60^{\circ} \mathrm{C}\right)$ & $43.5 / 44.3$ & \\
\hline & 2966Water $\left(27^{\circ} \mathrm{C}\right)$ & 44.7 & \\
\hline \multirow{2}{*}{$5 \mathrm{DBT}$} & 300 Octane $\left(27 / 60^{\circ} \mathrm{C}\right)$ & $43.7 / 44.4$ & \multirow{4}{*}{5} \\
\hline & 2932Water $\left(27^{\circ} \mathrm{C}\right)$ & $44.7 / 45.0$ & \\
\hline \multirow{2}{*}{$5 \mathrm{DBTO}_{2}$} & 300 Octane $\left(27 / 60^{\circ} \mathrm{C}\right)$ & $43.7 / 44.5$ & \\
\hline & 2922Water $\left(27^{\circ} \mathrm{C}\right)$ & $44.7 / 45.0$ & \\
\hline \multirow{2}{*}{ 20DBT } & 300 Octane +3900 Water & $46.8 \times 46.8 \times 93.6 /$ & \multirow{4}{*}{20} \\
\hline & $\left(27 / 60^{\circ} \mathrm{C}\right)$ & $47.4 \times 47.4 \times 94.8$ & \\
\hline \multirow{2}{*}{$20 \mathrm{DBTO}_{2}$} & 300 Octane +3900 Water & $47.0 \times 46.9 \times 93.7 /$ & \\
\hline & $\left(27 / 60^{\circ} \mathrm{C}\right)$ & $47.4 \times 47.4 \times 94.6$ & \\
\hline
\end{tabular}




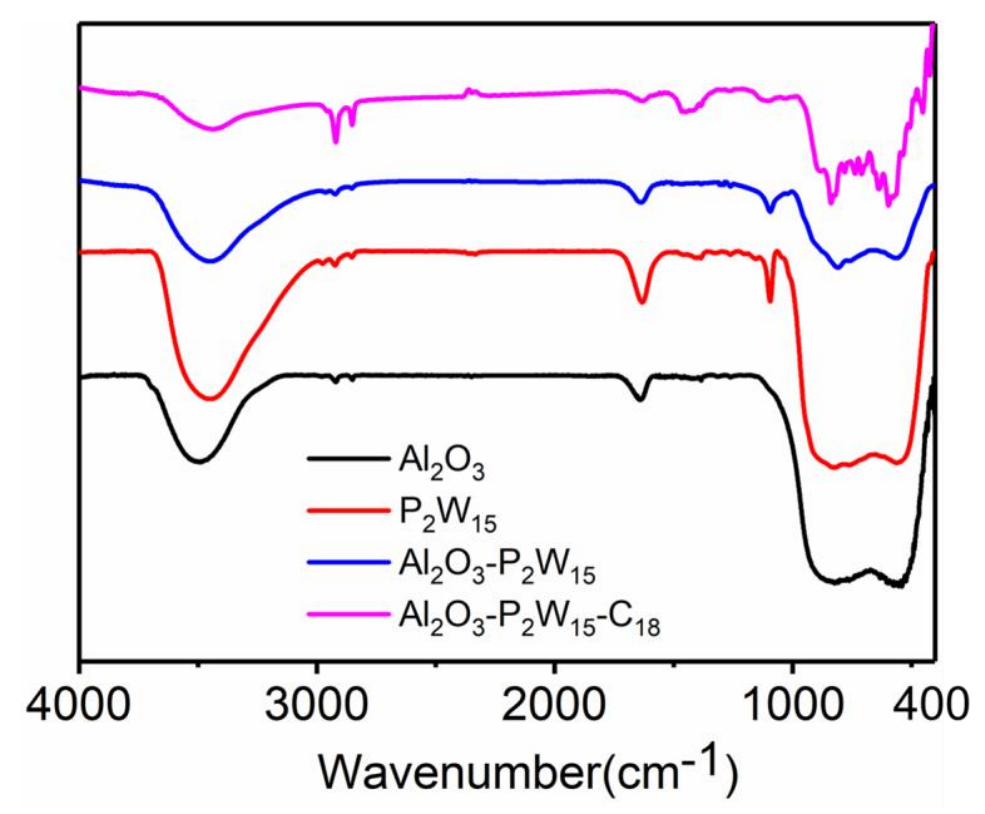

Figure S1. FTIR spectra of $\mathrm{Al}_{2} \mathrm{O}_{3}, \mathrm{P}_{2} \mathrm{~W}_{15}, \mathrm{Al}_{2} \mathrm{O}_{3}-\mathrm{P}_{2} \mathrm{~W}_{15}$ and $\mathrm{Al}_{2} \mathrm{O}_{3}-\mathrm{P}_{2} \mathrm{~W}_{15}-\mathrm{C}_{18}$.

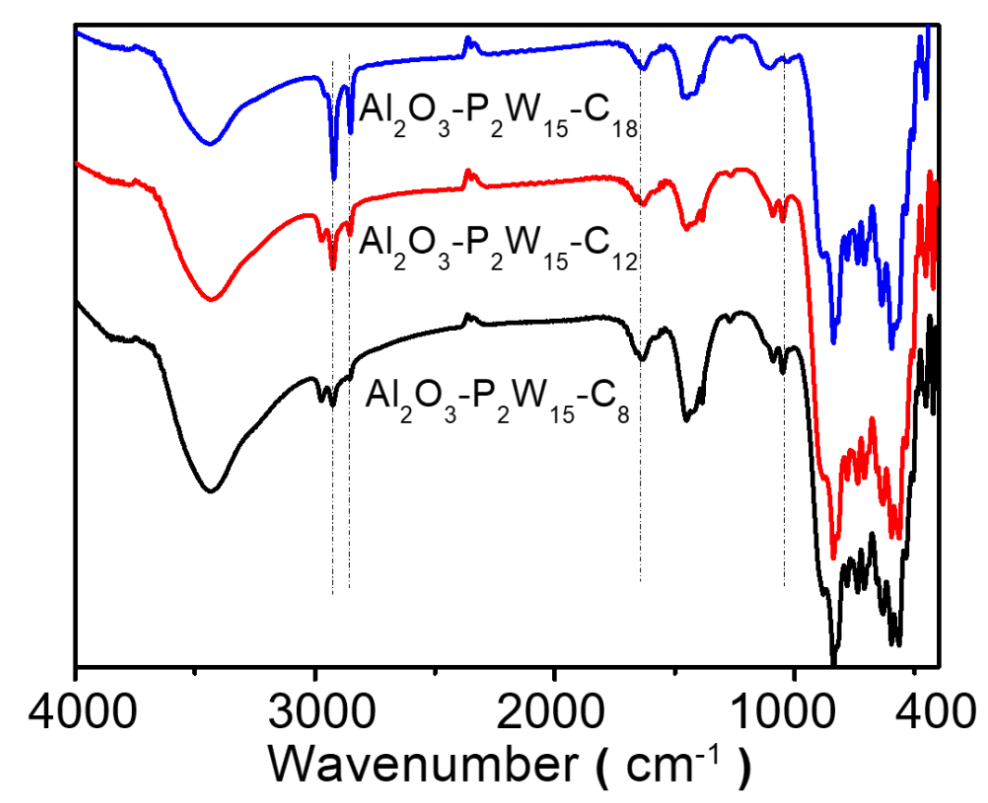

Figure S2. FTIR spectra of $\mathrm{Al}_{2} \mathrm{O}_{3}-\mathrm{P}_{2} \mathrm{~W}_{15}-\mathrm{C}_{8}, \mathrm{Al}_{2} \mathrm{O}_{3}-\mathrm{P}_{2} \mathrm{~W}_{15}-\mathrm{C}_{12}$ and $\mathrm{Al}_{2} \mathrm{O}_{3}-\mathrm{P}_{2} \mathrm{~W}_{15}-\mathrm{C}_{18}$. 

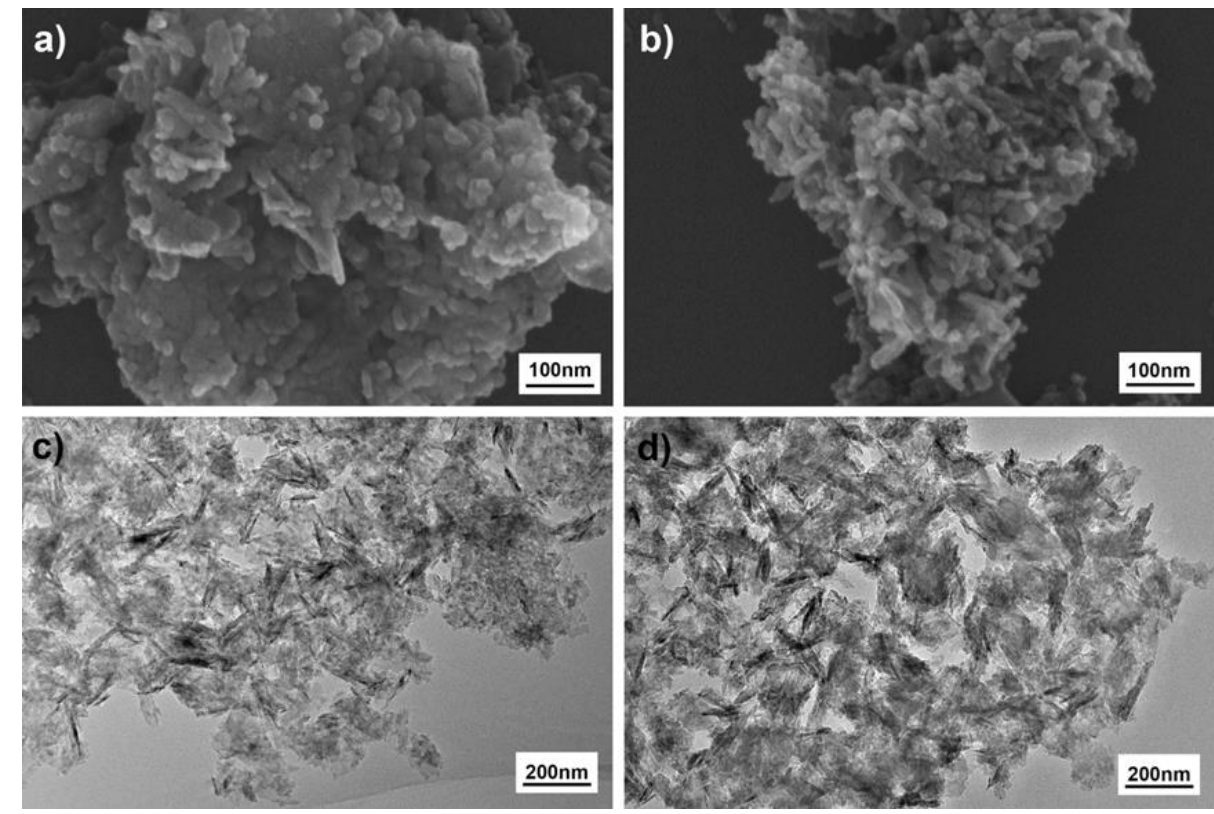

Figure S3. SEM image (a) and TEM image (c) of $\mathrm{Al}_{2} \mathrm{O}_{3}-\mathrm{P}_{2} \mathrm{~W}_{15}-\mathrm{C}_{8}$. SEM image (b) and TEM image (d) of $\mathrm{Al}_{2} \mathrm{O}_{3}-\mathrm{P}_{2} \mathrm{~W}_{15}-\mathrm{C}_{12}$.

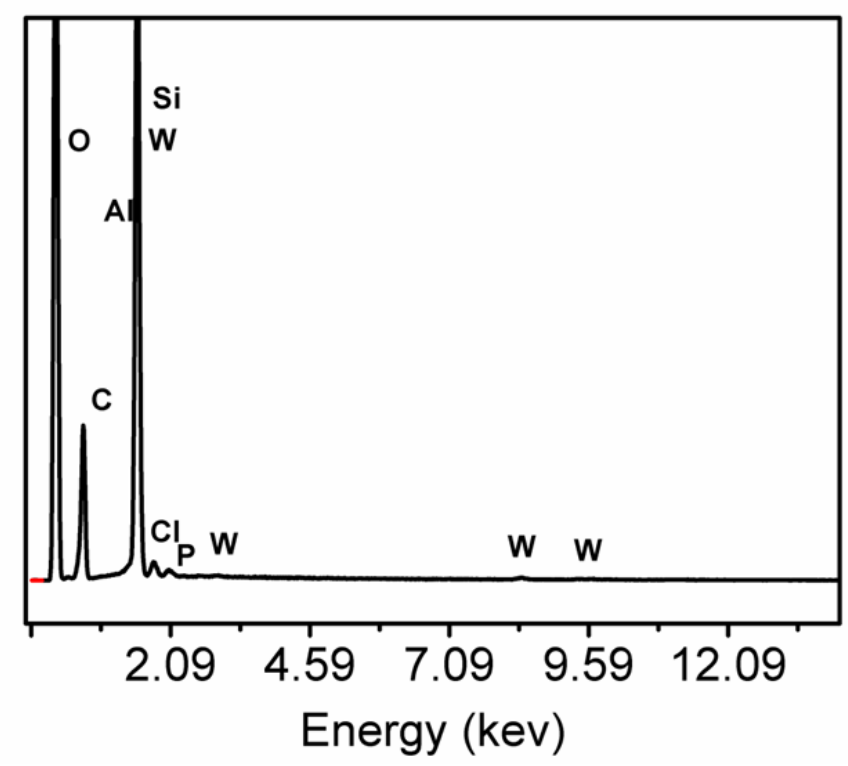

Figure S4. SEM-EDS of $\mathrm{Al}_{2} \mathrm{O}_{3}-\mathrm{P}_{2} \mathrm{~W}_{15}-\mathrm{C}_{18}$. 
Table S2. Textural properties of $\gamma-\mathrm{Al}_{2} \mathrm{O}_{3}, \mathrm{Al}_{2} \mathrm{O}_{3}-\mathrm{P}_{2} \mathrm{~W}_{15}$ and $\mathrm{Al}_{2} \mathrm{O}_{3}-\mathrm{P}_{2} \mathrm{~W}_{15}-\mathrm{C}_{18}$ catalysts.

\begin{tabular}{c|ccc}
\hline & Surface area $\left(\mathbf{m}^{\mathbf{2}} / \mathbf{g}\right)$ & $\begin{array}{c}\text { Pore volume } \\
\left(\mathbf{c m}^{\mathbf{3}} / \mathbf{g}\right)\end{array}$ & $\begin{array}{c}\text { Pore diameter } \\
(\mathbf{n m})\end{array}$ \\
\hline$\gamma-\mathrm{Al}_{2} \mathrm{O}_{3}$ & 56.57 & 0.34 & 29.15 \\
$\mathrm{Al}_{2} \mathrm{O}_{3}-\mathrm{P}_{2} \mathrm{~W}_{15}$ & 44.11 & 0.21 & 28.34 \\
$\mathrm{Al}_{2} \mathrm{O}_{3}-\mathrm{P}_{2} \mathrm{~W}_{15}-\mathrm{C}_{18}$ & 41.79 & 0.17 & 27.95 \\
\hline
\end{tabular}
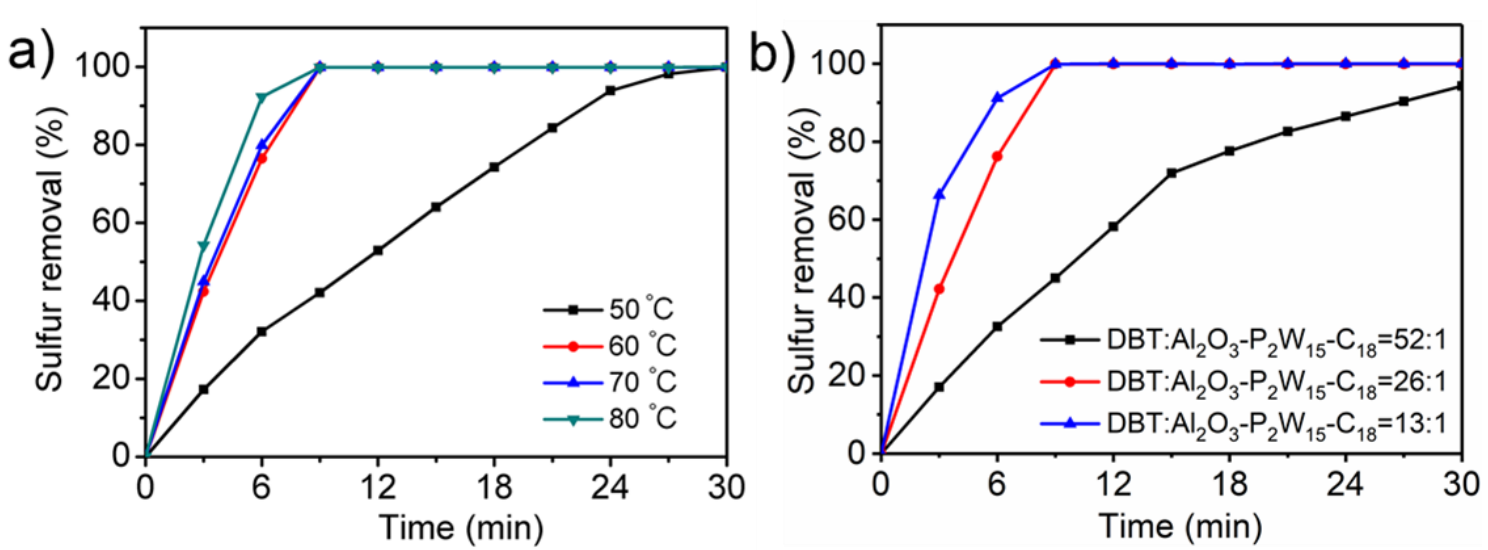

Figure S5. (a) Effect of temperature on sulfur removal of DBT, Reaction conditions: $\mathrm{H}_{2} \mathrm{O}_{2}: \mathrm{S}=3$, model oil $=5 \mathrm{~mL}, \mathrm{~S}=1000$ ppm, Catalyst: $\mathrm{Al}_{2} \mathrm{O}_{3}-\mathrm{P}_{2} \mathrm{~W}_{15}-\mathrm{C}_{18}, \mathrm{DBT}: \mathrm{Al}_{2} \mathrm{O}_{3}-$ $\mathrm{P}_{2} \mathrm{~W}_{15}-\mathrm{C}_{18}=26: 1$. (b) Effect of the amount of catalyst on sulfur removal of DBT, Reaction conditions: $60{ }^{\circ} \mathrm{C}, \mathrm{H}_{2} \mathrm{O}_{2}: \mathrm{S}=3$, model oil $=5 \mathrm{~mL}, \mathrm{~S}=1000$ ppm, Catalyst: $\mathrm{Al}_{2} \mathrm{O}_{3}-\mathrm{P}_{2} \mathrm{~W}_{15^{-}}$ $\mathrm{C}_{18}$. 

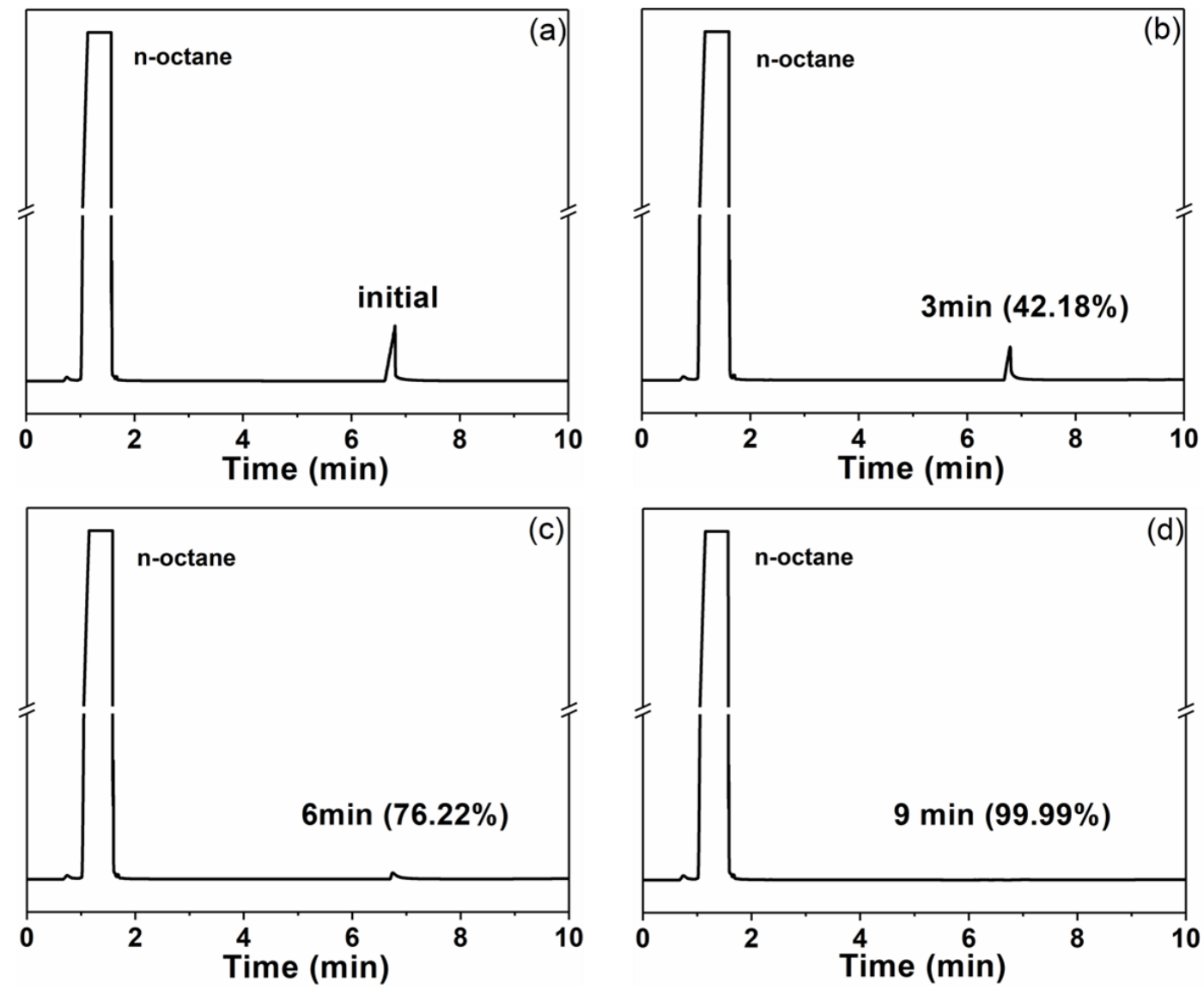

Figure S6. The influence of reaction time on DBT removal measured by GC-FID analysis.

Reaction conditions: $60^{\circ} \mathrm{C}, \mathrm{H}_{2} \mathrm{O}_{2} / \mathrm{S}=3$, model oil $=5 \mathrm{~mL}, \mathrm{~S}=1000 \mathrm{ppm}, \mathrm{DBT} / \mathrm{P}_{2} \mathrm{~W}_{15}=26: 1$, Catalyst: $\mathrm{Al}_{2} \mathrm{O}_{3}-\mathrm{P}_{2} \mathrm{~W}_{15}-\mathrm{C}_{18}$. 

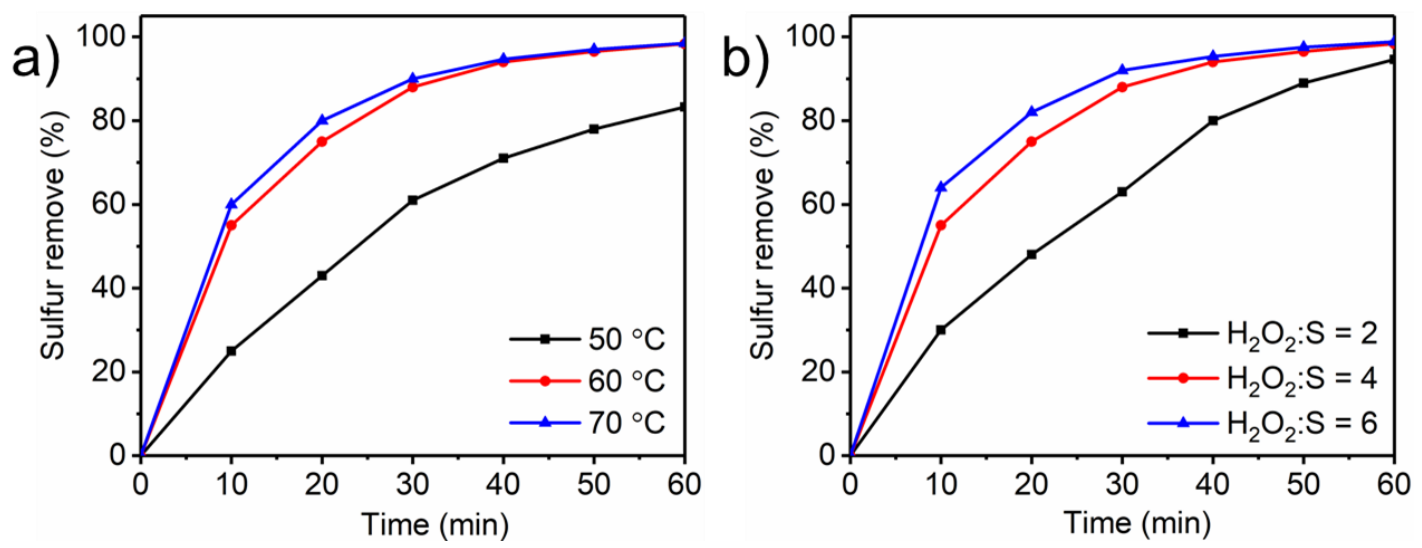

Figure S7. (a) Effect of temperature on sulfur removal of diesel fuel, Reaction conditions: $\mathrm{H}_{2} \mathrm{O}_{2}: \mathrm{S}=4$, S=425 ppm, Catalyst: $\mathrm{Al}_{2} \mathrm{O}_{3}-\mathrm{P}_{2} \mathrm{~W}_{15}-\mathrm{C}_{18}$. (b) Effect of the amount of $\mathrm{H}_{2} \mathrm{O}_{2}$ on sulfur removal of diesel fuel, Reaction conditions: $60{ }^{\circ} \mathrm{C}, \mathrm{S}=425 \mathrm{ppm}$, Catalyst: $\mathrm{Al}_{2} \mathrm{O}_{3}$ $\mathrm{P}_{2} \mathrm{~W}_{15}-\mathrm{C}_{18}$. 
Table S3. Comparison of catalytic oxidative desulfurization of real diesel and gasoline fuels by different immobilized catalytic systems (reported in literature) and the $\mathrm{Al}_{2} \mathrm{O}_{3}-\mathrm{P}_{2} \mathrm{~W}_{15}-\mathrm{C}_{18}$ from the present study.

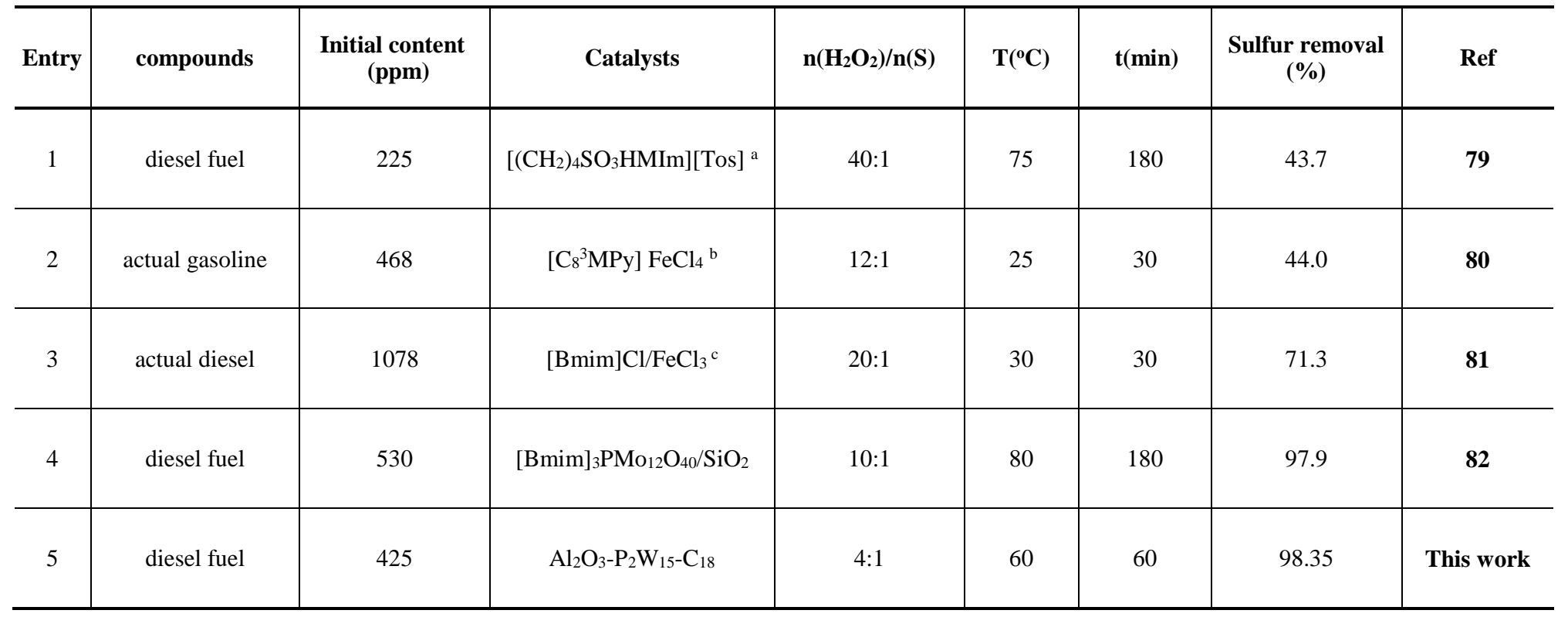

a. [( $\left.\left.\mathrm{CH}_{2}\right)_{4} \mathrm{SO}_{3} \mathrm{HMIm}\right][\mathrm{Tos}]$ : butyl-3-methylimidazolium p-toluenesulfonate; b. [C $\left.{ }^{3} \mathrm{MPy}\right] \mathrm{FeCl}$ : dialkylpyridinium tetrachloroferrates; c. Bmim: 1-butyl-3-methylimidazolium. 


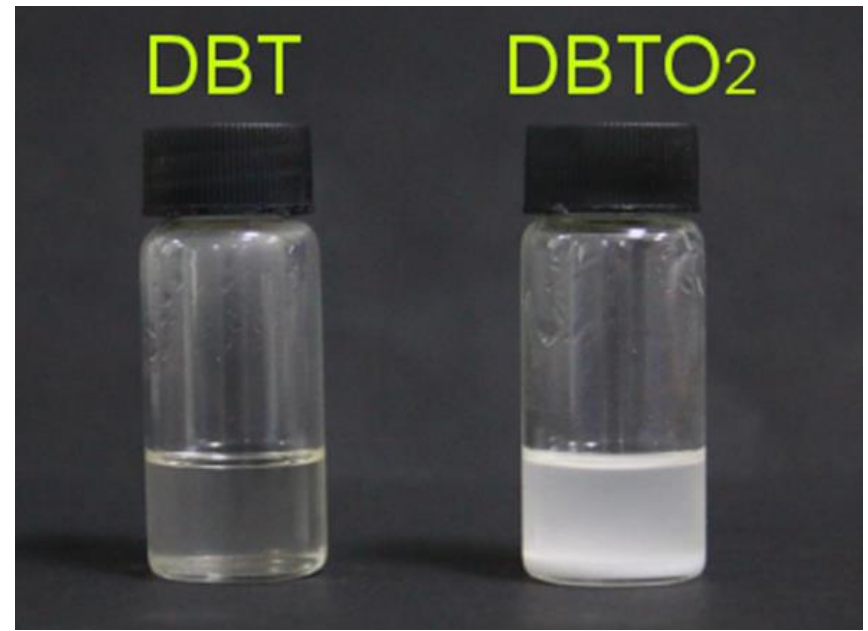

Figure S8. The image of solubility about $\mathrm{DBT}$ and $\mathrm{DBTO}_{2}$ in octadecyltrichlorosilane. 